\title{
Video Article \\ Super-resolution Imaging of the Natural Killer Cell Immunological Synapse on a Glass-supported Planar Lipid Bilayer
}

\author{
Peilin Zheng $^{\star^{1,2}}$, Grant Bertolet ${ }^{*^{1,2,3}}$, Yuhui Chen ${ }^{1,2}$, Shengjian Huang ${ }^{1,2}$, Dongfang Liu ${ }^{1,2,3}$ \\ ${ }^{1}$ Center for Human Immunobiology, Texas Children's Hospital \\ ${ }^{2}$ Department of Pediatrics, Baylor College of Medicine \\ ${ }^{3}$ Department of Pathology and Immunology, Baylor College of Medicine \\ * These authors contributed equally
}

Correspondence to: Dongfang Liu at dongfanl@bcm.edu

URL: https://www.jove.com/video/52502

DOI: doi:10.3791/52502

Keywords: Immunology, Issue 96, Natural killer cells, immunological synapse, imaging, STED, supported lipid bilayer, super-resolution

Date Published: 2/11/2015

Citation: Zheng, P., Bertolet, G., Chen, Y., Huang, S., Liu, D. Super-resolution Imaging of the Natural Killer Cell Immunological Synapse on a Glasssupported Planar Lipid Bilayer. J. Vis. Exp. (96), e52502, doi:10.3791/52502 (2015).

\section{Abstract}

The glass-supported planar lipid bilayer system has been utilized in a variety of disciplines. One of the most useful applications of this technique has been in the study of immunological synapse formation, due to the ability of the glass-supported planar lipid bilayers to mimic the surface of a target cell while forming a horizontal interface. The recent advances in super-resolution imaging have further allowed scientists to better view the fine details of synapse structure. In this study, one of these advanced techniques, stimulated emission depletion (STED), is utilized to study the structure of natural killer (NK) cell synapses on the supported lipid bilayer. Provided herein is an easy-to-follow protocol detailing: how to prepare raw synthetic phospholipids for use in synthesizing glass-supported bilayers; how to determine how densely protein of a given concentration occupies the bilayer's attachment sites; how to construct a supported lipid bilayer containing antibodies against NK cell activating receptor CD16; and finally, how to image human NK cells on this bilayer using STED super-resolution microscopy, with a focus on distribution of perforin positive lytic granules and filamentous actin at NK synapses. Thus, combining the glass-supported planar lipid bilayer system with STED technique, we demonstrate the feasibility and application of this combined technique, as well as intracellular structures at NK immunological synapse with super-resolution.

\section{Video Link}

The video component of this article can be found at https://www.jove.com/video/52502/

\section{Introduction}

The immunological synapse (IS) lies at a critical junction for cell activation and function ${ }^{1}$. It is the primary medium by which antigen presentation and cell-mediated immunity are carried out. The earliest microscopic studies of synapse formation utilized a cell-cell conjugate system ${ }^{2}$. The major limitation with this approach is that most of the conjugates will be viewed 'in profile', as it were, thus restricting the observer's view of the synaptic structure itself. In 1999, the Dustin laboratory addressed this limitation by utilizing the glass-supported lipid bilayer (SLB) technique ${ }^{3}$, which had been pioneered earlier by the McConnel laboratory ${ }^{4,5}$. This approach disposed of antigen presenting cells (APCs) in favor of a glass-supported planar lipid surface, into which proteins could be attached and move freely in two dimensions. Using this method, Dustin and colleagues were able to peer directly up into the synapse using high resolution fluorescence microscopy, and for the first time get a "face-to-face" look at the structure of the IS.

With the use of SLB system, the detail with which the IS can be visualized has been restricted only by the limitations of current imaging techniques ${ }^{6-8}$. Using standard illumination techniques, the minimum resolution (i.e., minimum distance between two distinct objects wherein they can be distinguished) has been $<200 \mathrm{~nm}$ on the basis of Rayleigh's criterion ${ }^{9}$. This limit hinders the imaging of very fine, molecular-scale structures that make up the synapse, and until the development of super-resolution imaging techniques ${ }^{10-12}$, visualization of these structures was confined to imaging of fixed cells using electron microscopy.

With the recent advent of a variety of super-resolution techniques, such as SIM (structured illumination microscopy), PALM (photoactivated localization microscopy), STORM (stochastical optical reconstruction microscopy), and STED ${ }^{10-12}$, investigators are now able to study these synaptic structures in unprecedented detail, which has in turn provided an increasingly clarified understanding of the IS. The advantages of STED microscopy have been described before ${ }^{13}$. Here we describe super-resolution imaging with STED microscopy equipped with the newlydeveloped $660 \mathrm{~nm}$ depletion laser. Compared to the conventional $592 \mathrm{~nm}$ depletion laser, the $660 \mathrm{~nm}$ laser allows for a broader selection of fluorescent dyes (see http://nanobiophotonics.mpibpc.mpg.de/old/dyes/), especially these red fluorophores.

Other publications have described the STED imaging of NK cell synapses on antibody-coated glass slides ${ }^{13,14}$. Here, the SLB system is combined with super-resolution STED microscopy to study the NK cell synapse. This technique has the advantage over antibody-coated slides 
of being a fluid mosaic, in which the embedded surface proteins can move freely in a flat two-dimensional surface ( $\mathrm{x}$-y plane). This more faithfully mimics the organic and mobile surface of a target cell, and consequently better recapitulates the formation of a physiologically relevant immune synapse.

The goal of this protocol is to provide the end-user with a detailed description of how to image the immunological synapse of NK cells by combining the SLB system and super-resolution STED microscopy. It will provide the end user with the steps necessary to: prepare the liposomes, construct protein-embedded bilayers, determine the protein density on the lipid bilayers, and acquire super-resolution images using STED microscopy. These techniques are not limited to the field of immunology, and can be broadly utilized across a variety of disciplines.

\section{Preparation of Liposomes}

1. Calculate the amount of chloroform-suspended stock solutions of 1,2-dioleoyl-sn-glycero-3-phosphocholine (DOPC) and 1,2-dioleoylsn-glycero-3-phosphoethanolamine-N-cap biotinyl (Biotin-PE) to make diluted stocks at the desired final concentration. To make final concentrations of $400 \mu \mathrm{M}$ DOPC and $80 \mu \mathrm{M}$ Biotin-PE phospholipids at $10 \mathrm{ml}$ each, start by placing $629 \mu \mathrm{l}$ of $10 \mathrm{mg} / \mathrm{ml} \mathrm{DOPC}$ and $88 \mu \mathrm{l} 10$ $\mathrm{mg} / \mathrm{ml}$ Biotin-PE into separate glass chromatography tubes.

NOTE: it is important to clean glass Hamilton syringes and glass chromatography tubes by cleaning solution (1 L $95 \%$ ethanol to $120 \mathrm{ml}$ water containing $60 \mathrm{~g}$ potassium hydroxide, $\mathrm{KOH}$ ), while transferring chloroform-suspended stock solution of DOPC and Biotin-PE.

2. Dry the chloroform with a stream of argon in the chemical hood. Seal the chromatography tube by parafilm.

3. Subject the newly dried liposomes to a high vacuum in a lyophilizer $\mathrm{O} / \mathrm{N}$ to remove any residual chloroform. For same-day completion, dry for $60-90 \mathrm{~min}$.

4. While the lyophilizer runs, prepare some dilution buffer. For this protocol, prepare $25 \mathrm{ml}$ consisting of $25 \mathrm{mM} \mathrm{Tris,} \mathrm{pH} 8.0 ; 150 \mathrm{mM} \mathrm{NaCl}$; and $2 \%$ (by weight) n-octyl- $\beta$-D-glucopyranoside $(O G)$ detergent. Mix the first two ingredients together first, then displace oxygen with argon before adding the dry OG powder. After preparation, filter the OG solution with 0.2 micron cellulose acetate membrane, and store at $4^{\circ} \mathrm{C}$.

5. In addition, prepare two screw-top bottles of $1 \mathrm{~L}$ of Tris-saline buffer at the same concentrations, but without OG. Place a distilled watercleaned magnetic stir bar in the bottom of each. Prepare 6 additional liters of the Tris-saline buffer. Remove oxygen from all the bottles with argon and place them at $4^{\circ} \mathrm{C}$ as well.

6. After lyophilization, dissolve the dried lipids in the Tris-saline OG buffer to make a $4 \mathrm{mM}$ solution of each. Following the example volumes, add $2 \mathrm{ml}$ to the DOPC tube, and $0.2 \mathrm{ml}$ to the Biotin-PE tube.

7. Mix together the biotin-PE lipids with the DOPC lipids. This improves the mobility of the SLB, as the coupled biotin can impair the fluidity of the phosphate head groups. To make a final concentration of $80 \mu \mathrm{M}$ Biotin-PE, mix $0.2 \mathrm{ml}$ of $4 \mathrm{mM}$ Biotin-PE and $1 \mathrm{ml}$ of $4 \mathrm{mM}$ DOPC. Then add $8.8 \mathrm{ml}$ of the Tris-saline OG buffer.

8. For a final concentration of $400 \mu \mathrm{M} \mathrm{DOPC}$, simply mix $1 \mathrm{ml}$ of $4 \mathrm{mM}$ DOPC with $9 \mathrm{ml}$ of Tris-saline OG.

9. Fill the sonicator with ice water. Put the glass tube containing the diluted phospholipid in the center of the sonicator by using a utility clamp. Sonicate the diluted phospholipid for 10 min until the solution becomes clear. NOTE: Add ice into the sonicator water bath to keep the temperature low since sonication will generate heat.

10. Fill in the tubes with argon to displace the oxygen in the air above the liquid, and seal them with parafilm.

\section{Dialysis of Liposomes}

1. Cut two sections of dry dialysis tubing (Molecular Weight cut-off: $12-14,000$, diameter: $6.4 \mathrm{~mm}$ ) of appropriate length (in this example, $40 \mathrm{~cm}$ ), one for each phospholipid dilution, from the roll.

2. Rehydrate the tubing sections by allowing them to soak in a $200 \mathrm{ml}$ of distilled water in a glass beaker for $2 \mathrm{~min}$.

3. Microwave this for 5 min at a high setting, or at least until the water comes to a boil.

4. Tie a knot at one end of each tube and rinse out the interior with a few milliliters of Tris-saline-OG buffer. Thereafter, meticulously squeeze out as much of this wash buffer as possible to minimize the amount of buffer remaining inside.

5. In a laminar flow hood, add the diluted phospholipids into each tube and clamp the open ends with a small dialysis tube closure so as to exclude all air. Complete air exclusion will require the sacrifice of a small volume of the sample by clamping below the "water line".

6. Immerse the samples in the prepared previously bottles of Tris-saline buffer without OG. Displace oxygen in the bottle with argon before resealing and place to stir $\mathrm{O} / \mathrm{N}$ at $4^{\circ}$.

7. Transfer the tubing into a new bottle of Tris-saline buffer without OG every $12 \mathrm{~h}$ for at least 3 times.

8. Shortly before removal of the dialyzed lipids, prepare a number of small tubes into which to aliquot the lipids by filling each with argon to displace the oxygen.

9. After $36 \mathrm{~h}$, take the dialysis bottles into the laminar flow hood and remove the dialysis tubes from the bottles. Have a bench diaper or beaker on hand to collect the wet runoff.

10. Cut the dialysis tubing above the clip, then remove the clip and carefully transfer the dialyzed lipid solution via pipet into $1 \mathrm{ml}$ aliquots in preprepared tubes filled with argon gas on ice.

11. Aliquot the aqueous liposome solution, use the argon stream to displace oxygen again in each tube

12. Store the liposomes at $4^{\circ}$. Do not freeze.

\section{Determination of Antibody Density on the Lipid Bilayer}

1. Prepare a dilution series of biotinylated, fluorescently-labeled antibody, $50 \mu \mathrm{l}$ in volume for each dilution, at the following concentrations: $0 \mathrm{nM}$ (blank), $10 \mathrm{nM}, 50 \mathrm{nM}, 100 \mathrm{nM}$, and $500 \mathrm{nM}$. (Hereafter referred to as "sample series").

2. Add $1 \mu \mathrm{l}$ of silica beads into 6 wells of a 96 -well v-bottom plate. Be sure to shake the beads well before pipetting, as they tend to settle. NOTE: If having ordered dry beads rather than suspended, follow the manufacturer's instructions to dilute. 
3. To these beads, add $2 \mu \mathrm{l}$ of mixed DOPC:Biotinyl phospholipids at a 1:1 ratio. Do this for each well.

4. Pulse the plate on a vortexer at medium strength 3 times for $10 \mathrm{sec}$ each to encourage interaction with the beads and the phospholipids.

5. Add $150 \mu \mathrm{l}$ of $5 \%$ casein to each well. Mix well by pipetting up and down three times.

6. Let the plate incubate in the casein solution for $10 \mathrm{~min}$, then wash it out. To wash, fill each well to a total volume of $250 \mu \mathrm{l}$ with HEPES buffered saline (HBS) with $1 \%$ human serum albumin (HSA). Centrifuge at 1,000 x g for 2 min. Pipette out and discard the top $200 \mu \mathrm{l}$ of supernatant, and repeat twice for a total of three wash cycles.

7. Add $50 \mu \mathrm{l}$ of streptavidin at a $333 \mathrm{ng} / \mathrm{ml}$ concentration. Pulse the plate again $3 \times 10 \mathrm{sec}$, and let it sit on a shaker for $15 \mathrm{~min}$. Wash $3 \times$ as in step 6 to remove unbound streptavidin.

8. Add $50 \mu \mathrm{l}$ of the fluorescently labeled biotinylated antibody from the previously prepared dilution series (see step 3.1) to each well, and replace the plate on the shaker for 20-30 min. Wash $3 x$ as in step 6 to remove unbound antibody.

9. After the final wash, resuspend the beads in $100 \mu \mathrm{l}$ of HBS/1\% HSA, then transfer to a FACS tube. Repeat this twice to ensure the efficient removal of all beads from the well, for a total of $300 \mu \mathrm{l}$, and do this for each well.

10. Bring the resulting tubes to the flow cytometer. It is time to read them.

11. Add 1 drop from the bottle marked "B" from the fluorescence intensity calibration (FIC) bead kit (hereafter referred to as "standard series") to a FACS tube, and dilute with $300 \mu \mathrm{l}$ of HBS/1\% HSA.

12. Read this tube, but do not record the data just yet. For now, simply ensure that the blank beads are properly zeroed. Make a histogram showing the fluorescence of the beads measured in the appropriate channel, then shift the voltage of the excitation laser down until the peak is at the far left side of the histogram.

13. Remove the tube and add 1 drop of each of the other 4 tubes in the series (labeled 1 through 4 ) to the same tube. Now, place the tube in the machine again, and record the resulting data, which should appear as 5 distinct peaks.

14. Read each individual tube from the sample series.

15. Using FACS analysis software, draw a gate spanning the entire width of each peak in the standard series histogram at the half-maximal point. That's one gate for each peak. Do the same for each sample. Note the MFI (mean fluorescence intensity) for each gate.

16. Using a spreadsheet program input the measured MFI values in the appropriate place. Also input the MESF (Molecules of Equivalent Soluble Fluorochrome) values (average number of fluorescent molecules coating each bead) for each bottle in the standard series. This information can be found by following the directions on the plastic jar the tubes came in.

17. Use the spreadsheet will plot the MFI against the MESF values, creating a linear correlation between the number of fluorophores and the measured intensity.

18. Use the module of 'proteins \& labels' in the micro-volume spectrophotometer to determine the ratio of dye to protein in the labeled antibody.

19. Input the labeling efficiency of the sample protein, the average diameter of the beads coated with lipids, and the MFI value for each entry. The spreadsheet will automatically use the formula from the line graph generated in step 3.16 to compute the MESF values for each protein dilution and the seeding density of the sample protein at each concentration.

\section{Isolating and Culturing Human NK Cells}

1. Aliquot $15 \mathrm{ml}$ peripheral blood or buffy coat into a $50 \mathrm{ml}$ conical tube. Dilute this blood with PBS containing $1 \%$ FBS at the ratio of $1: 1$.

2. Add $13 \mathrm{ml}$ of Ficoll gently to the bottom of the tube with a $10 \mathrm{ml}$ serological pipette.

3. Centrifuge this tube for $20 \mathrm{~min}$ at $1,200 \mathrm{xg}$ with the accelerator and the break off or at their lowest settings.

4. After centrifugation, use a serological pipette to collect the floating cloudy white middle layer of peripheral blood mononuclear cells (PBMCs), which should sit and the intersection between a clear yellow upper layer and a more cloudy pale-colored lower layer, both of which sit above a lowermost layer of red blood cells (RBCs). NOTE Do not to collect any RBCs in collecting the PBMCs.

5. Place the collected PBMCs in a new $50 \mathrm{ml}$ conical tube, and dilute to capacity with PBS containing $1 \%$ FBS. Centrifuge again, this time with the brake and accelerator on maximum, for $5 \mathrm{~min}$ at $300 \mathrm{xg}$.

6. Discard the supernatant and resuspend the cells in $10 \mathrm{ml}$ of PBS containing $1 \%$ FBS.

7. Count the cells while centrifuging once more at the same settings as in step 4.6.

8. Discard the supernatant once more, and resuspend the cells in R10 medium at a density of 10 million cells $/ \mathrm{ml}$.

9. Take 30 million cells in a $5 \mathrm{ml}$ polystyrene tube, and isolate NK cells using a magnetic separation kit, following the manufacturer's instructions. Following isolation, count the cells one more and resuspend at a density of $500,000 \mathrm{cells} / \mathrm{ml}$ in R10 complete medium (88\% RPMI; $10 \%$ FBS; $1 \%$ HEPES; $1 \%$ sodium pyruvate) supplemented with IL-2 (100 U/ml). Culture at $37^{\circ}$ in a $\mathrm{CO}_{2}$ incubator, and replace medium 2-3 times weekly.

\section{Assembling the Glass-supported Planar Lipid Bilayer}

1. Prepare $100 \mathrm{ml}$ piranha solution by mixing $30 \%$ hydrogen peroxide with sulfuric acid at a ratio of $1: 3$ in a beaker. NOTE: Always carry out work with noxious agents like sulfuric acid in a properly designated chemical fume hood.

2. Into this solution, immerse 2 rectangular \#1.5 coverslips in piranha solution for 20-30 min. NOTE: It is essential to clean the coverslips by piranha solution.

3. While the coverslips are being cleaned, take 1 tube of previously prepared $400 \mu \mathrm{M}$ DOPC lipids and 1 tube of previously prepared $80 \mu \mathrm{M}$ Biotin-PE lipids. Transport them on ice to the argon tank.

4. Displace oxygen in a new microcentrifuge tube with argon, then add together the DOPC and Biotin-PE at a 1:1 ratio. Specific volume will vary based on experimental needs, but should be at minimum $2 \mu \mathrm{l}$ each. Displace oxygen in the mixture tube once more with argon gas, and the individual reagent tubes as well, before returning the latter to the refrigerator.

5. After they are finished cleaning, thoroughly rinse the coverslips with distilled water. Set the coverslips out to air dry for a few minutes.

6. Withdraw $1.5 \mu \mathrm{l}$ of the liposome mixture prepared in step 5.4 and aliquot it in a single drop into one of the lane chambers of the chamber slide. The use of 2 drops per lane is typical, but not necessary.

7. Quickly and efficiently place the dry coverslip over the droplets. Ensure that the drops are sufficiently spaced so that they do not merge once the coverslip is placed. Furthermore, make sure that the drops remain circular and well-defined, without touching the edges of the chamber walls. Press down firmly in between and around each lane to ensure a watertight seal between the coverslip and slide. 
8. Mark the positions of the drops using a marker pen

9. Pass $100 \mu \mathrm{l}$ of aqueous $5 \%$ casein through the chamber to block the bilayer. Try to make sure that there are no bubbles in the flow chamber.

10. Inject $100 \mu \mathrm{l}$ of streptavidin at a concentration of $333 \mathrm{ng} / \mathrm{ml}$ into each lane. Incubate for 10-15 min at RT. Thereafter, wash by running $3 \mathrm{ml}$ of $\mathrm{HBS} / 1 \% \mathrm{HSA}$ through each lane to remove the excess streptavidin.

11. Add $100 \mu \mathrm{l}$ of biotinylated fluorescently labeled anti-CD16 such as Alexa Fluor 568 at the protein concentration previously determined to be most effective in section 3. Incubate in the dark for 20-30 min. Wash again by running $3 \mathrm{ml}$ of HBS/1\% HSA through each lane.

12. Flow $100 \mu \mathrm{l}$ of D-biotin at a concentration $25 \mathrm{nM}$ through the chamber in order to bind any excess streptavidin and thus eliminate the chance of non-specific binding of streptavidin to the cells.

13. Count NK cells and resuspend at a concentration of $500,000 / \mathrm{ml}$ in $\mathrm{HBS} / 1 \% \mathrm{HSA}$.

14. While spinning down the cells, wash the D-biotin out of the chamber with another $3 \mathrm{ml}$ of HBS/1\% HSA per lane.

15. Check the mobility of ligands on the SLB by fluorescence recovery after photobleaching (FRAP) on a total internal reflection fluorescence (TIRF) or confocal microscopy prior to adding NK cells.

16. Once the cells have finished spinning and have been resuspended at the desired concentration, add $100 \mu$ lo each lane.

17. Place the chamber in a $37^{\circ} 5 \% \mathrm{CO}_{2}$ incubator for 30-60 min.

18. After this incubation period, fix the cells with $4 \%$ paraformaldehyde at RT for $10-20 \mathrm{~min}$. Wash by running $3 \mathrm{ml}$ of PBS through each lane to remove the paraformaldehyde.

19. Add $400 \mu \mathrm{l}$ blocking buffer ( $5 \%$ normal donkey serum and $0.2 \%$ Tritron X-100 in PBS). Incubate at RT for $30 \mathrm{~min}$.

20. Stain F-actin and perforin by adding $200 \mu \mathrm{l}$ of diluted fluorescently-labeled phalloidin ( $1 \mathrm{unit} / \mathrm{ml}$ labeled phalloidin) and fluorescently-labeled anti-perforin $\mathrm{mAb}(500 \mathrm{ng} / \mathrm{ml}$ anti-perforin $\mathrm{mAb})$. Incubate at RT for $1 \mathrm{hr}$.

21. Wash by running $3 \mathrm{ml}$ of PBS. The chamber is ready for the imaging.

\section{Imaging of the NK Synapse on Lipid Bilayer using STED}

1. Turn on all necessary hardware modules.

2. Start up the image analysis software. Enable both resonant scanning and STED modules. After making these selections, wait for about 3-5 min for the software to initiate.

3. Click on the "Configuration" tab at the top of the screen.

4. Select "Laser Config" then turn on the white light and STED $592 \mathrm{~nm}$ lasers.

5. Choose the 100x objective, and align the excitation laser beam with the $592 \mathrm{~nm}$ depletion laser.

6. Select the "Laser Config" module, turn off the 592 depletion laser, and turn on the $660 \mathrm{~nm}$ depletion laser.

7. Place the slide upon the stage, over the lens. Bring the cells bound on the demarcated bilayer region into focus using the white light lamp and the oculars.

8. Return to the "Acquisition" tab, directly to the right of the "Configuration" tab.

9. Click the "Switch to Whitelight" tab, then turn that module on and drag the excitation laser line to the appropriate wavelength.

10. Select the desired detector from the list of those available, then set the detection range to encompass the appropriate range of wavelengths. NOTE: NEVER put the detection range directly beneath the excitation beam.

11. Click on the "Seqential" button in the left-hand "Acquire" toolbar to bring up the Sequential Scanning dialogue in the bottom of the left-hand toolbar. This allows the user to add multiple sequences, each with a different excitation beam for a different color. Click "Between Frames", and then set the excitation frequency, detector, and detection range for each additional color as in steps 6.9 and 6.10 .

12. Once all the settings are optimized, hit "Start" to begin the acquisition process.

13. Apply the free deconvolution software (Huygens) using a setting designed for STED deconvolution, as described previously ${ }^{13}$.

\section{Representative Results}

Figure 1 shows the result of the antibody density on the lipid bilayer. The principle is to use standard beads to make the standard curve of MESF versus MFI via flow cytometry (A). The MFI of the sample series was converted into MESF using the standard curve. The antibody density on the lipid bilayer is linearly correlated with the antibody concentration (B). Figure 2 show the triple-color STED image of NK synapse on glass-support planar lipid bilayer. Anti-CD16 antibody on the lipid bilayer accumulates, triggering F-actin formation and the polarization and penetration of perforin through the F-actin mesh at the focal panel of immunological synapse in NK cells. Using this combined approach, one can cleanly observe the microclusters of fluorescently labeled anti-CD16 within the SLB, which directly mirrors the clustering of CD16 on the NK cell. Compared to the conventional confocal image, the structure of CD16 central cluster is more readily discerned in the STED image due to the depleted ambient fluorescence. Furthermore, the ultrastructure of the actin cytoskeleton is seen with significantly improved resolution. Consistent with previous observations $^{16,17}$, the perforin-positive lytic granules are seen positioned over regions of low F-actin density in the STED image, a crucial detail which is mostly lost in the confocal image. 
A



B

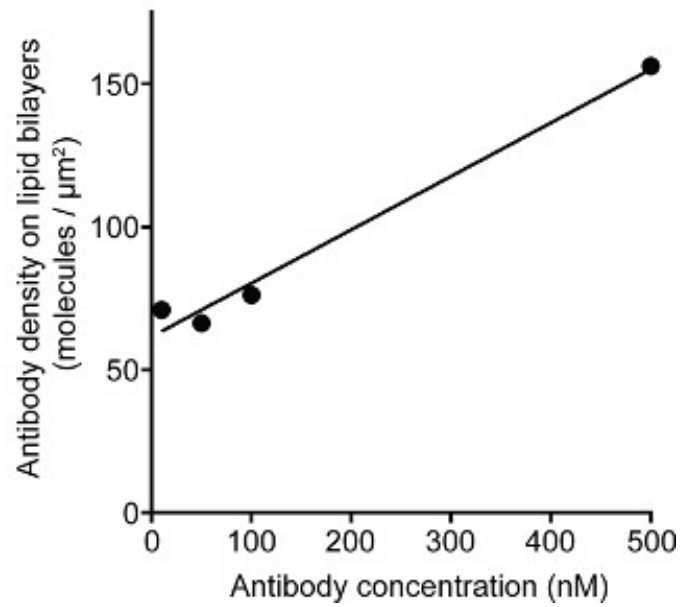

Figure 1. Density of $\mathbf{3 G 8}$ antibody on lipid bilayer. (A) Linear correlation between MESF and MFI for standard series. (B) Linear correlation between protein density and concentration for sample protein dilution series, showing the number of fluorescently-labeled protein monomers per unit area as a function of increasing concentration on lipid-coated silica beads.
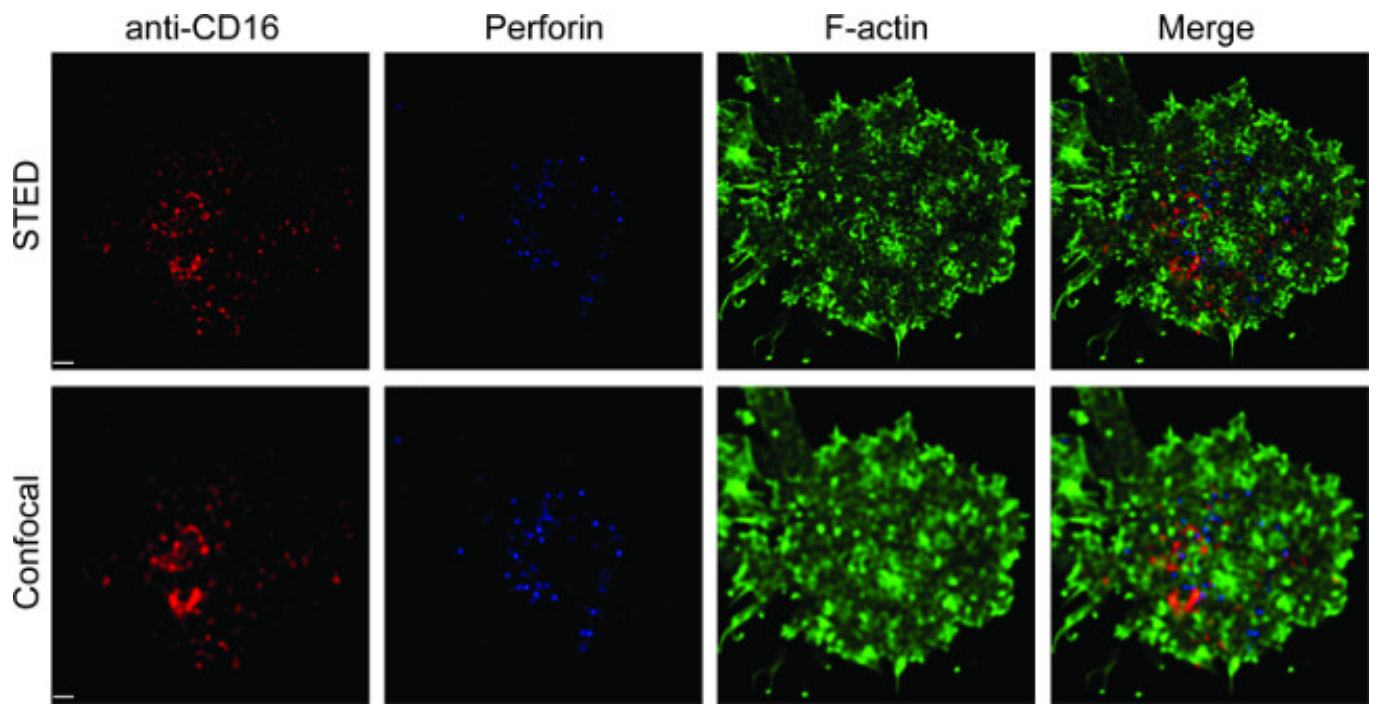

Figure 2. STED imaging of NK synapse on planar lipid bilayer. Primary NK cells were stimulated on the SLB containing biotinylated fluorescently labeled anti-CD16 (red), fixed, permeabilized, and then stained with phalloidin (blue) and anti-F-actin (green). An individual cell was first imaged under the normal confocal setting, and then the STED setting. Confocal and STED images were deconvoluted using Huygens software. Scale bar, $1 \mu \mathrm{m}$. Click here for a larger version of this figure.

\section{Discussion}

The novelty of the current study is that it combines the SLB technique with STED to study NK cell synapses. Previous studies have imaged the lipid bilayer with TIRF to study T cell synapse formation ${ }^{8}$ and signaling molecule trafficking on the plasma membrane ${ }^{6}$. Others have described STED imaging of NK cell synapses using antibody-coated glass slides ${ }^{13,14}$. The hybrid method described herein further builds upon these efforts by imaging the NK cell synapse with the enhanced clarity afforded by super-resolution imaging on the lipid bilayer surface, which better models the dynamic surface of an APC.

Although SLBs are artificial membranes lacking of cytoskeleton, lipid rafts, and other ligands that actual target cells or APCs possess, this technique can recapitulate important features such as the mobility and orientation of ligands. This allows the SLB system to serve as a reductionist approach in dissecting the contribution of individual receptors and ligands to formation of the IS and the dynamics of IS. The most important feature of SLBs is that researchers can combine this technique with high-resolution imaging approaches, such as confocal and TIRF microscopy. The introduction of STED microscopy even further increases this advantage, providing unprecedented insights into IS research and its clinical applications.

One potential criticism of this system is that the SLB does not adequately mimic the complex surface of an APC, thus giving rise to potentially non-physiological anatomical features in the resulting synapses. While it is true that the limited repertoire of surface molecules on the SLB does not fully recapitulate the heterogeneously populated surface of an APC, this limit can also be advantageous in that it allows investigators to determine the influence of individual receptor and ligand interactions on synapse formation. 
There are several crucial steps in the process. Among the most critical is that oxidation of the liposomes be prevented through constantly using argon to displace the oxygen in the tube and solution, such as in steps 1.10, 2.6, and 2.11. Oxidation of the lipids will result in decreased lipid mobility, thus impeding the ability of surface proteins to move freely and participate in synaptic structuring. Likewise, it is also crucial to remove all the chloroform in the liposome by lyophilization (step 1.2). In the determination of protein density in the lipid bilayer, it is of importance to first disperse the silicon beads into a homogenous suspension free from clusters. If necessary, sonication of beads can be applied. In the assembling the SLB, the early steps (5.1-5.8) wherein the coverslips are cleaned, the drops are placed, and coverslip is affixed are vital. A mistake in any of these can necessitate starting the experiment over (from the beginning of section 5). For this reason, it is good practice to clean more coverslips than will be needed to save time in case of a mishap.

Non-clustering is the most frequent issue when working with this system. If, when visualizing the cells in the final step, one fails to find any fluorescent synapses, there are a few steps that can be taken. Another stain for the cognate cell-surface receptor can be added to the chamber to verify that the cell has not formed a synapse with the bilayer, While cells can adhere non-specifically to the glass coverslip or bilayer surface, synaptically-involved surface proteins should appear as distinct clusters at the plane of the cell-bilayer interface, whereas unengaged surface proteins should appear as diffuse staining around the perimeter of the cell. Should this method fail, one should check their cells via flow cytometry to ensure that the particular marker one is hoping to study is expressed in adequate abundance on the cell surface. Certain surface proteins are known to be down-regulated over long term in vivo culture.

While this protocol details specifically how to visualize NK cell synapse formation, the SLB system can be used to study synapse formation in any immunocyte imaginable simply by substitution of the primary ligand in step 5.11. Multiple ligands may also be added simultaneously. One also need not use a streptavidin-biotin system for adhering the surface proteins within the bilayer. Nickel-NTA:histidine interactions are also viable. However, due to the high strength and specificity of the streptavidin:biotin interaction, our lab prefers this system. One may also vary the concentration of cells added onto the bilayer from the prescribed density in step 5.13, as well as the duration of the subsequent incubation period in order to observe synapses at different stages of maturation. This can even be done live, although this of course excludes the possibility of visualizing intracellular structures (unless they are already labeled with a fused fluorescent tag; our lab uses a few such altered cell lines). Due to the high degree of customization possible in this protocol, one can use the basic SLB technique, along with STED imaging, to address an incredibly diverse range of questions in immunology, cell biology, and biochemistry, including basic lipid dynamics ${ }^{15}$, synapse formation ${ }^{16}$, intracellular signaling ${ }^{17}$, and tumor cell metastasis ${ }^{18}$.

\section{Disclosures}

The authors have nothing to disclose.

\section{Acknowledgements}

We thank Emily Mace and Malini Mukherjee for imaging and deconvolution analysis. Work in the Liu laboratory was supported in part by the Baylor-UTHouston Center for AIDS Research Core Support Grant number Al36211 from the National Institute of Allergy and Infectious Diseases, the Caroline Wiess Law Fund for Research in Molecular Medicine, Texas Children's Hospital Pediatric Pilot Research Fund, and the Lymphoma SPORE Developmental Research Program from Baylor College of Medicine and the Methodist Research Institute.

\section{References}

1. Dustin, M. L., Long, E. O. Cytotoxic immunological synapses. Immunol. Rev. 235, (1), 24-34 (2010).

2. Monks, C. R., Freiberg, B. A. K. upferH., Sciaky, N., Kupfer, A. Three-dimensional segregation of supramolecular activation clusters in T cells. Nature. 395, (6697), 82-86 (1998).

3. Grakoui, A., et al. The immunological synapse: a molecular machine controlling T cell activation. Science. 285, (5425), 221-227 (1999).

4. Hafeman, D. G., von Tscharner, V., McConnell, H. M. Specific antibody-dependent interactions between macrophages and lipid haptens in planar lipid monolayers. Proc Natl Acad Sci USA. 78, (7), 4552-4556 (1981).

5. Tscharner, V., McConnell, H. M. Physical properties of lipid monolayers on alkylated planar glass surfaces. Biophys J. 36, (2), 421-427 (1981).

6. Crites, T. J., Chen, L., Varma, R. A TIRF microscopy technique for real-time, simultaneous imaging of the TCR and its associated signaling proteins. J. Vis. Exp. (2012).

7. Prins, K. C., Vasiliver-Shamis, G., Cammer, M., Depoil, D., Dustin, M. L., Hioe, C. E. Imaging of HIV-1 envelope-induced virological synapse and signaling on synthetic lipid bilayers. J. Vis. Exp. (2012).

8. Vardhana, S., Dustin, M. Supported Planar Bilayers for the Formation of Study of Immunological Synapses and Kinapse. J. Vis. Exp. (2008).

9. Michalet, X., Weiss, S. Using photon statistics to boost microscopy resolution. Proc. Natl. Acad. Sci. U. S. A. 103, (13), 4797-4798 (2006).

10. Huang, B., Bates, M., Zhuang, X. Super-resolution fluorescence microscopy. Annu. Rev. Biochem. 78, 993-1016 (2009).

11. Lippincott-Schwartz, J., Manley, S. Putting super-resolution fluorescence microscopy to work. Nat. Methods. 6, (1), 21-23 (2009).

12. Hell, S. W. Far-field optical nanoscopy. Science. 316, (5828), 1153-1158 (2007).

13. Mace, E. M., Orange, J. S. Visualization of the immunological synapse by dual color time-gated stimulated emission depletion (STED) nanoscopy. J. Vis. Exp. (2014).

14. Rak, G. D., Mace, E. M., Banerjee, P. P., Svitkina, T., Orange, J. S. Natural killer cell lytic granule secretion occurs through a pervasive actin network at the immune synapse. PLoS Biol. 9, (9), e1001151 (2011).

15. Leutenegger, M., Ringemann, C., Lasser, T., Hell, S. W., Eggeling, C. Fluorescence correlation spectroscopy with a total internal reflection fluorescence STED microscope (TIRF-STED-FCS). Opt Express. 20, (5), 5243-5263 (2012).

16. Liu, D., Bryceson, Y. T., Meckel, T., Vasiliver-Shamis, G., Dustin, M. L., Long, E. O. Integrin-dependent organization and bidirectional vesicular traffic at cytotoxic immune synapses. Immunity. 31, (1), 99-109 (2009). 
17. Liu, D., Peterson, M. E., Long, E. O. The adaptor protein Crk controls activation and inhibition of natural killer cells. Immunity. 36, (4), 600-611 (2012).

18. Wu, J. C., et al. Antibody conjugated supported lipid bilayer for capturing and purification of viable tumor cells in blood for subsequent cell culture. Biomaterials. 34, (21), 5191-5199 (2013). 